\title{
Ethics and Etiquette in Humanitarian Engagement-101
}

\author{
Jackie L. Clark, Ph.D. ${ }^{1}$
}

\begin{abstract}
As the travel industry continues to grow, so does the creation and proliferation of voluntourism opportunities offered to individuals who want to impact the lives of populations due to adversities or misfortunes of war, weather, or poverty. A more popular form of tourism for individuals to volunteer professional or personal expertise in a chartable manner is often termed "voluntourism." Unquestionably, there is a lure to volunteer for a short-term experience in exotic lands with the hopes of improving living conditions. This article aims to identify how an individual can move from being a well-meaning voluntourist to an engaged and dedicated humanitarian by following professional ethical principles and etiquette behavior.
\end{abstract}

KEYWORDS: ethics, etiquette, voluntourism, ethnocentrism, autonomy, justice, beneficence, maleficence, cultural differences

The human desire to lighten a little the torments of all these poor wretches... creates a kind of energy which gives one a positive craving to relieve as many as one can.

Henri Dunant, humanitarian aid worker and founder of the International Committee of the Red Cross. ${ }^{1}$

Recently, the literary world has focused on the failings in humanitarian efforts. To reach that conclusion, one only has to look at current literary titles such as "Crisis Caravan: What's Wrong with Humanitarian Aid?"2; "Humani- tarianism in Question ..."3; "Toxic Charity ..."4; "A Bed for the Night: Humanitarianism in Crisis"5; "When Helping Hurts"; and "Dead Aid: Why Aid is Not Working ... in Africa." Some critics suggest that humanitarian aid has not only failed, but in fact has left those living in low- and middle-income regions in worse condition than prior to any assistance. ${ }^{8}$ Such criticism is often based on the proliferation of naive benevolent notions in saving the (inaptly named) "third world"/"undeveloped world" due (in part) to philanthropic organizations humanitarian service provision that may include, in part or sum, grossly abundant
${ }^{1}$ Callier Center, University of Texas at Dallas, Dallas, Texas.

Address for correspondence: Jackie L. Clark, Ph.D., Callier Center, University of Texas at Dallas, 1966 Inwood Road, Dallas, TX 75235

(e-mail: jclark@utdallas.edu).
Humanitarian Audiology; Guest Editor, Steven P. Smith, Au.D.

Semin Hear 2020;41:83-91. Copyright (C) 2020 by Thieme Medical Publishers, Inc., 333 Seventh Avenue, New York, NY 10001, USA. Tel: +1(212) 760-0888. DOI: https://doi.org/10.1055/s-0040-1708526. ISSN 0734-0451. 
financial profit; pervasive ethnocentrism; reinforcement of the practice of paternalism; misrepresentation and/or over generalization of actual sociopolitical situations in low- or middle-income countries; and lastly ambiguity of ethical practices during deployments. In fact, the situation has become significant enough that some countries have issued bans on receiving certain aspects of humanitarian service provision.

As the travel industry grows by leaps and bounds, "voluntourism" has been more prevalently offered by specialized tourist companies to diligently connect individuals with excess discretionary funds who want to make a difference with those who struggle just to survive in a low-resourced area. In fact, the pursuit of voluntourism is endorsed in the travel industry, and also by various global philanthropic organizations. Consequently, without adequate planning and preparation, there are abundant opportunities for humanitarians to engage in catastrophic missteps and contradictory actions that oppose the primary mission of improving the lives of individuals living in low-resource regions. As the adage says: Become part of the solution not part of the problem. The humanitarian/voluntourist should continuously aspire to be part of a contextually based solution by taking on the challenge of learning the nuances of local infrastructure and utilizing existing resources while keeping in mind possible resource shortages. Any unfamiliar ethics and etiquette should be strongly examined and considered while serving in an unfamiliar location. The unavoidable fact is that: with each deployment, a voluntourist is imposing themselves into a community and for better or worse, they are (negatively and/or positively) influencing the ecosystem of each community in which they are engaged. As witnessed through news media, the need is tremendous while the supplies are diminished or absent in most low- and middle-income areas. A humanitarian's hope during each engagement should be to ensure that any humanitarian footprint is prominently marked with collaborative long-term contextual solutions, resolutions with positive outcomes rather than a proliferation of problems, conundrums, and backward movement. The purpose of this article is to reduce the number of voluntourists while empowering and increasing humanitarians who may be considering or are answering a calling to provide ethical ear and hearing care services in low- and middleincome areas. Volumes have been and continue to be written about various aspects of humanitarian deployments; this article will focus on two foundational components: ethics and etiquette for positive humanitarian works.

\section{FOUNDATIONAL ETHICS}

Ethical practice is a viable means of ensuring that public safety and dignity are kept at the forefront of all (off- and on-shore) on-site hands on experience (i.e., engagements). To a novice, there may be confusion between the terms "morality" and "ethics." Though these terms may appear to be synonymous, they are not.

Morality is often loosely thought of as a system of values and principles of conduct based on behaviors determined by society regarding what is right or wrong: good or bad. It is based on personal judgments and understandings about what one ought to do in a particular situation so that the community can live cooperatively and in harmony. Morality is not fixed; it can (and does) change over time depending on the desires of society.

Ethics is based on standards of behavior and defined as doing (action verb) or promulgating what is defined by an individual or a code of conduct determined by a group and is based on moral philosophy. However, it is worth noting that just as collective values change over time, so do decisions about what is considered ethically acceptable. It goes without saying, if one lacks a moral compass there will be no desire to behave within a code of conduct.

Some specific ethical principles for healthcare in the 21 st century are based on theories and applications in the health sector that specifically include nonmaleficence, beneficence, autonomy, and justice. Historically, many difficult healthcare decisions have been focused on these four specific foundational principles that will be discussed below with illustrative examples.

Nonmaleficence is based on the Hippocratic teaching of "first, do no harm." This concept would appear to be simple and straightforward. As healthcare practitioners, there is always a 
deep desire to do nothing that could be harmful to patients. However, ethicists would have us ponder the acceptable meaning of harm. In general terms, harm in audiology could include pain, discomfort, inconvenience, expense, omission of critical information, negligence, and/or violation of autonomy or disability. There are various ethical theories that impact nonmaleficence and a deeper meaning of harm, such as consequentialism, natural law, deontologism (ethics of duty), virtue, or egoism. These theories certainly have relevance in various healthcare sectors; however, for brevity, these will not be discussed in this article.

Example 1: In a voluntourism deployment, a well-meaning voluntourist, who is not in the construction trade, paid thousands of dollars for a short-term opportunity of painting walls and building houses or facilities in a low-/middleincome area. Little thought is put toward the economic harm on the local level by depriving the various local unemployed workers who could be easily compensated for the same tasks undertaken by a voluntourist-let alone funds spent in travel and lodging that could be utilized in a different manner. Hand tools and other materials, readily available locally, were purchased and transported from U.S. retail establishments. It could be argued that the local economy has been harmed even in the simple act of voluntourists' bringing tools that were purchased at retail establishments prior to arriving, thus denying financial benefit to local merchants.

Example 2: In a voluntourism deployment, an enthused humanitarian audiologist leads a team to conduct annual hearing screenings with the intention of providing 100 to 200 hearing aid fittings over a 5 -day period at no charge in a lowresourced area. Little to no thought is put toward partnering with the local audiologist whose services will be sought over time by the many hearing aid recipients who likely will have questions and concerns about hearing aid care and repairs. It is not unusual that the hearing aid recipients may have expectations that the local audiologist would also render free services. Consequently, the services offered by the local audiologist may be significantly devalued by the local community (resulting in harm). In addition, if the local audiologist refuses to accommodate the hearing aid recipient's request for free services, a harmful chasm could arise between recipients and local audiologist. Even the local recipients are harmed from the lack of ethics of duty by the absence of plans for continuity of care by the well-meaning voluntourists.

Beneficence is derived simply as an act of charity, mercy, and kindness-simply from the dictum of "doing good to others." Ultimately, an action is performed for the benefit of the recipient/patient, whose welfare and well-being are the ultimate goals of healthcare. Conversely, refraining from creating or causing harm would implicitly imply a benefit to the beneficiary of the act of charity, mercy, and/or kindness. It is expected that healthcare professional's role is one consistent with exhibiting acts of kindness and helpfulness.

Example 3: In a first-time voluntourism deployment, a voluntourist (who is a cochlear implant specialty audiologist) discovers a profoundly deaf nonverbal 9-year-old child in lowincome area with no hearing health care or deaf education specialists for hundreds of miles. Despite the limitations arising from the language differences, the voluntourist spoke to the parents regarding hearing loss and how their child would return with the ability to hear after minor surgery if the family agreed that their child could travel with the voluntourist the next day to the United States (at no cost to the family). The parents agreed with limited to no understanding about the surgery or the necessary long-term therapy associated with the free cochlear implant. Since the parents had no means of communicating with the child, it was clear on the day of departure that the child was visibly surprised and upset. Weeks and months later, when the parents were asked by their community members about their child, they simply answered that the doctor was insistent that their child would be well after surgery and would return with the ability to hear and speak as well as go to school. After an abundance of U.S. press releases within the Unites States, numerous articles, and live news interviews about the generosity of the voluntourist and workplace, more than adequate funds were raised to underwrite the cost of surgery, as well as for cochlear implant mapping, short-term speech therapy, and one-way airfare ticket to return the child home. 
When considering who benefitted the most in this example, though likely unintentional, clearly the voluntourist and workplace profited as a consequence of media promotion, increased patient base, and donation of funds in excess of the monetary targeted goal. It is uncertain what long-term benefit the child and family reaped from the extravagant gift made possible by the generous community and voluntourist. Little thought was given to other viable contextual communication options for the child, such as create or identify an existing local school for the deaf, where the child could have received ongoing education as well as emotional support from family and community. Instead the family has reaped a very short-term solution, and is unduly harmed with omission of critical information that there is neither local specialized therapy, equipment, long-term services for cochlear implant equipment, and cochlear implant mapping nor specialized medical support in the event of postsurgical complications.

Autonomy ensures that individuals have the capacity as agents in self-determination in decisions without the undue influence from others. In health matters, it is inferred that the individual has capacity to make informed, uncoerced decisions independently. Great care must be taken especially with underrepresented vulnerable populations (i.e., children, racial or ethnic minorities, those with language differences, emotional or mental handicaps, undereducated, underinsured, or socioeconomically disadvantaged, etc.) to avoid any risk of having autonomy deprived by either from naive, persuasive, or disingenuous individuals. Such risk multiplies exponentially in the instances of language and/or dialectical differences when contextual barriers cannot adequately be overcome to mitigate any degree of coercion. As a consequence, vulnerable populations experience power imbalances and may not have complete understanding of situations and procedures. This population may not understand that they have autonomy to refuse or accept procedures without recourse. While it is well understood that informed consent must be obtained within the realms of health care, it becomes a challenge to ensure that there is no perception of/coercion due to an assumption of wealthy and/or highly educated experts influencing the consent.
Continuation Example 3: Due to communication barriers, the nonverbal deaf 9-year-old was not provided the benefit of assent or refusal to leave the comfort and safety of family and home with a stranger, undergo surgery, and travel in a foreign (and likely unknown) mode of transportation. It is probable that the family succumbed to the power imbalances experienced through coercion by a wealthy and highly educated individual. If true autonomy were encouraged, there would be every effort to provide a thoughtful interpreter from the community who would be sensitive to the education level and dialect spoken by the family. After divulging all risks and benefits and long-term commitment by the family, there would be thoughtful dialogue (not a monologue by the voluntourist) that would also provide ample decision-making time for the entire family (including the child) to ensure that informed decision was obtained without duress or lacking information. Indeed, it would be important for the family to express understanding that a positive outcome of surgery is not guaranteed and that following surgery the family might need to move to another city or country to obtain the necessary therapy and medical follow-up. If the family and child's autonomous decision making in th earlier scenario is disregarded, the die is cast. Irrevocably lifelong harm has occurred to the child and family due to the lack of regard for individual autonomous decision making.

Justice is behavior that is concerned about fairness and equal treatment. This can be accomplished with practicing fair procedures and processes (procedural justice), and subsequently allocating available resources with equity and fairness (distributive justice). In the healthcare sector, the dilemma of justice often occurs especially within low- and middle-income countries when selecting which individuals will receive services within the context of severely limited resources.

Example 4: An inexperienced first-time voluntourist is deployed for the summer to an area that has no ear and hearing services within the province. As a passionate pediatric audiologist, the voluntourist initiated a newborn hearing screening program so that every child with hearing loss would be identified and fit with a gifted set of hearing aids. Within just a few 
months, 25 infants were fit with a set of the latest state-of-the-art hearing aids and earmolds. With a bit more reflection, there are additional questions also about beneficence, nonmaleficence, and autonomy in this scenario. However, when placing more focus on both procedural and distributive justice, it appears there was no engagement of community leadership in the decision making about resource allocation priorities. Granted, the supplies were brought to the community as a gift from the voluntourist; however, local policy leaders (who were not included in the decision) may have preferred to allocate all or part of the severely limited resources to the adults in the community. It is difficult to predict if the local policy leaders would have recommended allocation of resources to babies within the community to acquire oral language skills or for school children to succeed in the classroom, or parents/ adults to remain gainfully employed in the workforce so that the entire family is economically sustained. The local policy makers were not given an opportunity to articulate the preferred specific procedures used in determining how resources are distributed throughout the community (i.e., according to family financial needs, etc.). Consequently, such decisions that impact the community need to be deferred to local policy leaders who understand the local history and focus of healthcare priorities.

Much like peeling back the layers of an onion, one quickly realizes that each of the aforementioned principles of ethics can be complex and potentially confusing. Often times, well-meaning voluntourists engage in seemingly simple decisions that ultimately result in far-reaching ramifications long after the volunteer has departed from the community. Voluntourists may have every intention of committing to multiple visits to the site of deployment, but life and unforeseen circumstances can (and often do) prevent continued or sequential deployments. Consequently, an additional set of principles of sustainability are well paired with principles of ethics detailed earlier. The basic principles surrounding sustainability dictate that long-term successfully performing programs fully engage local leadership willing to fully invest and make critical local decisions within the context of known external and internal forces within the community. Principles are simply guidelines and cannot stand in isolation to solve problems. Ultimately, as moral agents, the humanitarian volunteer must look toward guidance by local authorities to make difficult decision for their own community. After all, it is unrealistic to expect communities to passively wait for outsiders to arrive annually to make noncontextual decision on behalf of a community.

\section{ETIQUETTE}

The relationships of trust that Volunteers build with ... the people they serve is what gives our Volunteers the understanding of the local context and the credibility to become catalysts of community-led change. We help our communities recognize the potential within themselves and build upon it for a brighter future.

Director Carrie Hessler-Radelet, at a Peace Corps training event, in Siem Reap, Cambodia, March 21, $2015^{9}$

Cross-cultural misunderstandings between providers and patients or between a community outsider and the community can lead to mistrust and frustration that ultimately can jeopardize future engagements. By integrating the principles of ethics, one is not guaranteed success, but it is an invaluable beginning in a humanitarian deployment. One positive first step of engaging a community as a committed and credible humanitarian, and not a voluntourist, begins with investing in lasting long-term friendships by understanding the stories within context of each community. Community trust is built upon awareness of the many cultural influences, such as concept of time and ethnocentrism (just to name a few).

Cultural awareness and sensitivity will ensure a more solid footing in those early steps of engagement. Etiquette is considered practiced codes of behavior that are well entrenched in each community's culture (Oxford English Dictionary). Indeed, culture is transmitted from one generation to another and is unconsciously and implicitly integrated. When 
mothers tell their children "behave yourself," it encompasses not only eating behaviors at the table but other social behaviors such as demonstrating respect for individuals in the community. A community is far more forgiving of one's own children learning to integrate these important social graces than an adult "outsider" from a high-income country who is presumed to be educated and wealthier than the local community.

Real, meaningful change in communities doesn't happen from the top down, it happens from the ground up. It happens when you build on the strengths that already exist in those communities. It happens when you empower the leaders that are already there, and then they go on to empower others.

Former First Lady Michelle Obama, at a Peace Corps training event, in Siem Reap, Cambodia, March 21, $2015^{9}$

Much like an iceberg, most cultural information hidden below the water is more difficult to discern, especially if one is not part of the community. However, much of the cultural information influences behaviors such as idea of fairness, importance of time, manner of addressing elderly individual, or someone of a different gender. The parts of the iceberg that are visible (above the water) are those behaviors that are easily observable, such as food, music, literature, religious rituals, style of dress, holiday customs. ${ }^{10}$

Example 5: A young, eager novice voluntourist wanted to make a positive first impression by continuously making direct eye contact while firmly shaking hands throughout the conversation with the middle-aged Tribal Chief. In some countries within the western hemisphere (where the young voluntourist lives), the well-accepted behavior of making direct eye contact throughout introductions and conversation is considered a sign of respect and attentiveness; the firm handshake between herself and the Tribal Chief was meant to demonstrate additional balance of power. Unfortunately, the novice voluntourist is unaware that the tribe's local etiquette of direct eye contact from a female to male, especially elder male leader, is a sign of grievous disrespect.
In the context of the Tribal Chief s customs, females should remain respectful of powers of authority who are males by continuously diverting their eyes downward with their heads bowed to demonstrate respect for the elderly male and deference to the immense power differences between them. It is noteworthy to mention that etiquette in some countries with the direct eye contact (whether from a male or female) is seen as a sign of a very dishonest and untrustworthy person, yet other countries consider a long gaze as an open sign of aggression. ${ }^{11,12}$

- Concept of time, in general, is seen in two polemic domains: monochronic and polychronic time. Monochronic concept of time is more consistently seen in North America and many parts of Europe. With this view, time is considered discreetly quantifiable and marked by succinct schedules and deadlines; promptness is looked upon favorably, and people adjust themselves and agenda to suit the schedule. Conversely, the concept of polychronic time, often seen in Africa and Central Asia, is considered relative and abundant. It can be adjusted to suit the needs of people, and may involve unrushed time at the beginning of a meeting (perhaps while sipping tea) talking about topical items such as vacations, families; multiple interruptions during a meeting are considered part of the norm. ${ }^{10}$

\section{Example 6: A voluntourist from the United} States who owns a very busy and profitable private hearing healthcare practice arrives 15 minutes prior to the appointment at the Provincial Minister of Health's office in an impoverished African nation. The purpose of the visit is to discuss making hearing health care a priority within the provincial health clinics. Unfortunately, the Minister of Health's Secretary tells the visitor that her boss is not in the office and is not certain if he will be in today. After waiting for an hour, the voluntourist becomes overtly angry and leaves while making loud comments about respecting people's time. This collision of two cultures with differing concepts of time happens frequently. As the guest in the Minister of Health's country, it should be expected that all concepts of time default to the local (in this case) polychronic view 
of the host. It is also important to remember that there is a power differential present, in which the Minister of Health who wields the greater power. Rather than becoming offended by his monochronic perception of tardiness for a meeting with a local leader from a polychronic society, the angry voluntourist can take advantage of the opportunity to embrace more time for reflection in the local polychronic environment.

- Ethnocentrism is the result of judging other cultures and communities according to values and standards of one's own culture and/or ethnic group. Often the judgments come from the belief that any other culture is inferior. ${ }^{10}$ It is not unusual to hear a novice traveler frequently make remarks, "At home we do everything so much better than 'they' do here." In fact, ethnocentrism is based on an individual's narrow experiences, and an unconscious bias that makes one favor what he or she feels is safe, familiar, and likeable. Consequently, one tends to make general assumptions for which we have limited understanding. Ethnocentrism can lead individuals to want to make every place just like it is at home. It can also lead an individual to make sweeping generalizations from an isolated event about an entire culture or community. As a product of its time, the opening quote of this article by the passionate and dedicated founder of the International Red Cross Committee is rife with ethnocentric sentiments. To reduce the inherent ethnocentric behaviors as humans, it becomes a challenge for all to question one's own basis of views and attitudes of the world as well as tolerance of cultural differences.

Example 7: After a conversation with a local nurse, a voluntourist asked about the method of maintaining patient records in the rural hospital. The local nurse's response is met with a remark that in the United States we use "our" computers in the hospital to document all patient encounters so that any hospital personnel can access the information and not send a patient home to retrieve their national health record card. Unfortunately, the voluntourist did not delve further to understand that frequent power outages occur throughout the region, and the rural hospital computers were 8-yearold donations from a sister hospital in the United States. It seemed that in addition to power outages, the computers crashed most frequently in the exceedingly humid and hot season. Rather than asking about proposed local solutions or learning how the hospital was able to effectively manage hard copy records within the context of the region, the voluntourist was looking to "fix" a presumed "problem" at the hospital within the context of a high-income country solution.

Additional consideration: ethical photography is the recognition of responsibly preserving dignity while also providing faithful depiction of the surroundings. In this age of selfies, voluntourists often do capture their act of good deeds. It is important for all who travel outside of the U.S. borders to know that photographing federal, provincial, and city structures (such as airports, hospitals, clinics) in most countries is a punishable crime. Rarely do we hear of such arrests, but a foreigner remains at the mercy of a tired, underpaid border authority, who may neither be humored by naive traveler's photographic antics nor be culturally flexible.

When humanitarians hold themselves to the same standards of contact in a professional role as in the United States, there would never be a doubt within HIPAA regulations of inappropriate acts of photography in the healthcare delivery realm. In fact, photography is rarely considered professionally necessary to demonstrate improvement of individuals who are deaf or hard of hearing. In addition, some consider photography of patients in low- and middleincome areas as a dehumanizing act that promotes the dichotomy of "us" and "them," and perpetuates the colonial mindset of the highincome countries "helping" or "fixing" the "problems of the poor."

Example 8: A freshly minted audiologist was given the graduation gift of travel, and chose to volunteer with an existing program that dispenses hearing aids in foreign countries. Not only was the entire team strongly encouraged to actively photograph their work activities, but there was one individual tasked with only taking photos and videos of the entire team's engagement. In fact, the most prized photos were of parents and or recipients openly weeping and 
demonstrating what was assumed their deep gratitude. It is understood that philanthropic organizations are deeply dependent on documenting the fruits (often through videos and photography) reaped by donations. ${ }^{2}$ However, if the primary aim of onsite photography is for promotion of a philanthropic organization, then beneficence is realized only by the philanthropic organization, and not within the provision of healthcare to the community. On occasion, voluntourists argue that recipients "welcome" the photography. In fact, when asked, the recipients have given informed consent and agreed to the photography. It will never be known if the agreement was due to the obvious power imbalance between the provider of hearing aids and the recipient with no financial means of purchasing the product if the response was "no" to the request of photography. When such a power differential exists, the principle of autonomy of recipient is questioned and evidence of paternalism surfaces. It is important to note that there is a difference between excessively taking opportunistic, dehumanizing, and emotionally laden photos of engagements versus taking nonemotionally laden photos of someone with whom you have a friendly relationship or a mutual success story that includes a distinct knowledge between the subject and taker of the photo about each other's history (including names and detailed information).

\section{CONCLUSION}

What I say is this, and this I do not say as an Englishman. God made us different, you and I, and your fathers and my fathers. For one thing, we have not the same notions of honesty and speaking the truth .... And look now what you do? You come and judge us by your standards of morality.

\section{Rudyard Kipling, East and West}

Of course, a humble humanitarian volunteer would be concerned about effectively navigating through the choppy waters of cultural differences and hopefully feel drawn to diligently anticipate and avoid cultural missteps. By no means should this listing of etiquette tips in humanitarian deployments be considered exhaustive. There are many additional steps involved in the pre-, during-, and postdeployment stages. This article describes only some of the many common cultural differences that may be encountered. One valuable resource that should always be considered is through a local cultural guide who will be on-site with the humanitarian team. Often, when there are language differences, a team leader will engage local interpreters. Not only is there a rich opportunity of building long-term relationships but also learning about the local history and cultural behaviors from the designated interpreters. Some other resources that should be considered include the following: Peace Corps (https://www.peacecorps.gov/stories/); "Audiology Without Borders" collection of articles that highlight various humanitarian programs within The Hearing Journal (http:// journals.lww.com/thehearingjournal); Coalition for Global Hearing Health's (CGHH) annual meeting of humanitarians worldwide (http:// coalitionforglobalhearinghealth.org).

The CGHH was officially began in 2009 and in 2010 gained not-for-profit status as an organization with the purpose to advocate for effective hearing health services and policies; to equip and empower hearing healthcare professionals, families, educators, communities and those with hearing loss; and to perpetuate best practices. In addition to annual conferences, one offering by the $\mathrm{CGHH}$ is the very newly compiled document, "Guidance for Best Practices for Humanitarian Hearing Care Outreach Projects" (http://coalitionforglobalhearinghealth.org). It was created to serve as a resource for individuals or groups who are considering providing sustainable and successful humanitarian ear and hearing care programs while also maintaining contextually based approach. The simply worded document is framed around the premise of collaboration and cooperation, and organized with the "Top 20 Typical Questions" from inexperienced volunteers who are considering entering the arena of humanitarian and/or community-based services.

It might appear from this article that volunteering skills for humanitarian purposes is foolhardy and unwelcome. Quite the opposite is true. Inarguably, the global need is great, as cited 
by the World Health Organization's estimation in 2012 that approximately $5.3 \%$ of the world's population experiences significant hearing loss, of which most are living in low- and middleincome countries typically with no or limited ear and hearing services available. ${ }^{13}$ Without a doubt, both the voluntourist and humanitarian will have life-changing enduring experiences and memories to relay long after returning from deployment. There is an over-abundance of short-term, ill-equipped voluntourists. Conversely, there is also a paucity of well-equipped volunteer humanitarians who desire to effectively serve by upholding high ethical standards and emotionally invest in locally led sustainable programs to attain lifelong relationships with communities. Ultimately, attaining cultural competence requires managing the dynamics of differences, constantly acquiring cultural knowledge, and adapting to cultural contexts of the communities without judgment. In contrast to the voluntourist, a well-equipped and effective volunteer humanitarian will strategically and positively contribute, and, in fact, make a difference contextually to the community. If one learns to relax and realize that some (hopefully small) missteps will occur along the road to cultural competence, the journey will be rewarding.

\section{CONFLICT OF INTEREST}

The author is a co-founder and current volunteer co-director of the Coalition for Global Hearing Health and receives no financial support.

\section{REFERENCES}

1. Dunant H. A Memory of Solderino, International Committee of the Red Cross; Geneva; 1959
2. Polman L. Crisis Caravan: What's Wrong with Humanitarian Aid? New York: Metropolitan Books: Henry Hold and Company; 2010

3. Barnett M, Weiss TG. Humanitarianism in Question: Politics, Power, Ethics. Ithaca: Cornell University Press; 2008:36-38

4. Lupton RD. Toxic Charity: How the Church Hurts Those They Help. New York: HarperOne; 2011

5. Rieff D. A Bed for the Night: Humanitarianism in Crisis. New York: Simon \& Schuster Paperbacks; 2002

6. Corbett S, Fikkert G. When Helping Hurts: How to Alleviate Poverty without Hurting the Poor and Yourself. Chicago: Moody Publishers; 2012

7. Moyo D. Dead Aid: Why Aid Is Not Working and How There Is a Better Way for Africa. New York: Farrar, Straus and Giroux; 2009

8. Sullivan N. Opinion: When volunteering abroad does more harm than good. Huffington Post. Available at: https://www.huffpost.com/entry/opinionsullivan-volunteering-abroad_n_5a7de894e4b044b 3821d1627? guccounter=1\&guce_referrer=aHR0c HM6Ly93d3cuZ29vZ2xlLmNvbS8\&guce_referrer_sig=AQAAAHe6bfRecVHlexWhplzqMYmD tdgMlBwE81jCCyhr2Woptq7fG6S-ULyuhAX2 R912F-4mtzc32SS239wQkvXWIaHvntL_FY51G L-DIMifE1QdQutV4kI_1ehAOswKcByzej0WfRu_p3wVjwgJWaAUBrU1sHwqtIdledCWa18D4 QUd. Accessed February 12, 2018

9. Obama M Speech at a Peace Corps Training Event. Siem Reap, Cambodia; March 21, 2015

10. Corps P. Culture Matters: Peace Corps Information Collection and Exchange. Peace Corps: 1999

11. Akechi H, Senju A, Uibo H, Kikuchi Y, Hasegawa T, Hietanen JK. Attention to eye contact in the West and East: autonomic responses and evaluative ratings. PLoS One 2013;8(03):e59312

12. Bright Hub Media. Making Eye Contact: What are you saying in various cultures? Available at: https:// www.brighthubeducation.com/social-studies-help/ 9626-learning-about-eye-contact-in-other-cultures/. Accessed September 1, 2019

13. World Health Organization. Prevention of Blindness and Deafness; Estimates. Available at: http:// www.who.int/pbd/deafness/estimates/en/2012. Accessed October 31, 2016 